\title{
12 Pax Mongolica, Handel, Sklaverei und der „Schwarze Tod“
}

Bedeutende Forscher haben entdeckt, daß es neben ,autochthonen', d.h. landgeborenen, auch ,autothalassische‘, d.h. rein vom Meere bestimmte Völker gegeben hat, die niemals Landtreter gewesen sind und die nichts vom festen Lande wissen wollten, als daß es die Grenze ihrer reinen Meeresexistenz war. ${ }^{1}$

Die Unterscheidung zwischen sog. Landtretern und - um der Diktion des zitierten Carl Schmitts (1888-1985), eines wichtigen, aber wegen seiner Haltung zum Nationalsozialismus auch sehr umstrittenen deutschen Staatsrechtlers und politischen Philosophen, zu folgen - „Seeschäumern“, hat viele Autorinnen und Autoren immer wieder beschäftigt. Bei der Betrachtung des Zeitraums zwischen 1280 und 1360 auf der Krim (und darüber hinaus) meint man fast, Schmitt hätte am Beispiel der Interaktionen zwischen der Goldenen Horde und den italienischen Protagonisten in der Schwarzmeerregion die Differenz zwischen Land- und Seevölkern besonders trefflich beschreiben können. Tatsächlich erwähnte er in seinem Werk „Land und Meer“ Venedig, pries nicht nur dessen Reichtum, sondern auch „die diplomatische Überlegenheit, mit der die Seemacht die Gegensätze zwischen den Landmächten auszunützen“ wusste. ${ }^{2}$ Das mächtige Mongolenreich hingegen blieb unerwähnt. Und dies sicher nicht ohne Grund, denn in der vergleichenden Forschung zur Rolle von Imperien in der Geschichte wird immer wieder darauf hingewiesen, dass Historiker und Historikerinnen maritime Großreiche über lange Zeit (und nicht immer überzeugend) für entwickelter und moderner hielten als kontinentale. Mittlerweile ist die Imperiumsforschung von diesem strikten Diktum abgewichen und bescheinigt auch imperialen „Landtretern" innovatives Potential. ${ }^{3}$ Wie sich zeigt, ist die Frage nach den italienischmongolisch-tatarischen Interaktionen und ihren Folgen für die Halbinsel Krim auch unter Einbeziehung der Kategorien ,Rückständigkeit‘ oder ,Erfolg‘ höchst interessant.

Tatsache ist, dass sowohl die vermeintlich modernen italienischen Stadtstaaten als auch die angeblich rückständige Goldene Horde über eine gewisse Zeit territorial sehr ausgedehnte, aber in ihren Funktionsweisen und Strukturen sehr unterschiedliche Herrschaftsentitäten aufbauen und erhalten konnten. Festzuhalten ist überdies, dass es ahistorisch wäre, diese in der Vormoderne entstan-

1 Schmitt C. (1981), 10.

2 Schmitt C. (1981), 20.

3 Jobst, u.a. (2008), hier 29.

Ә OpenAccess. (C) 2020 Kerstin S. Jobst, publiziert von De Gruyter. (cc))BY Dieses Werk ist lizenziert unter der Creative Commons Attribution 4.0 International. https://doi.org/10.1515/9783110520620-014 
denen Staatswesen mit unseren zeitgenössischen Maßstäben $\mathrm{zu}$ messen. ${ }^{4}$ Gleichwohl gab es Phänomene, die uns selbst im heutigen Licht als fortschrittlich oder effektiv erscheinen und die von dem ,rückständigeren' Kontinentalimperium etabliert wurden. Dazu zählen beispielsweise die in der Horde eingeführten Systeme der Besteuerung und Konskription sowie das Straßen- und Postwesen. ${ }^{5}$ Es gab weitere moderne Erscheinungen, für die die Mongolen verantwortlich zeichneten: Im Schwarzen Meer und damit eben auch auf der Krim existierte ab dem ausgehenden 13. und bis in die Mitte des 14. Jahrhunderts eine intensive Kontaktzone zwischen den Mongolen, die den eurasischen Raum kontrollierten, und den italienischen Stadtstaaten, die den Seehandel dominierten. Dieser Zeitraum wird ex post als „Pax Mongolica“ bezeichnet und umfasst ungefähr die Jahre 1280 bis 1360. Händler konnten in dieser Phase weitgehend sicher ihren Geschäften nachgehen - und besaßen damit einen immensen Aktionsradius, der letztlich von China bis weit nach Europa und Afrika reichte. Diese Sicherheit generierte der mongolische Staat bzw. (wie es wohl treffender ist) Staatenbund, der im Übrigen keineswegs eine Despotie nach unserem heutigen Verständnis war, ${ }^{6}$ stellte der Chan doch eher einen Primus inter pares dar. ${ }^{7}$ Ein Nebeneffekt der Pax Mongolica war die Verbreitung elaborierter (Kultur-)Techniken aus der Medizin, Mathematik oder Astronomie - und zwar von Osten nach Westen! ${ }^{8}$

Durch die allmähliche Desintegration des Mongolenreiches und den sich abzeichnenden Zerfall in einzelne Chanate - eines davon wurde schließlich das Krim-Chanat -, aber auch durch den Ausbruch der Pest, die sich durch den regen Austausch von Menschen und Waren schnell verbreiten konnte, endete die Pax Mongolica. Dies bedeutete aber nicht das Ende der italienischen Kolonien auf der Krim, welche sich noch ein gutes Jahrhundert, bis das Osmanische Reich sich 1475 Caffa einverleibte und die italienischen oder armenischen Kaufleute mehrheitlich nach Pera migrierten (Kapitel 15) bzw. in die Sklaverei gerieten, halten konnten. ${ }^{9}$

4 Zur grundlegenden Literatur zur Goldenen Horde zählen u.a. (immer noch - trotz der teilweise problematischen Terminologie) Spuler (1965); Fedorov-Davydov (1973); Weatherford (2004); Halperin (1987); Ostrowski (1998). Vor allen Dingen Ostrowski arbeitet sich sehr überzeugend an der in westlichen Diskursen äußerst dominanten Vorstellung der angeblichen Rückständigkeit der Horde ab.

5 Hartog L. (1996), $164 \mathrm{f}$.

6 Als sehr wirkungsmächtig für diese Auffassungen erwies sich das mehrfach neu aufgelegte Werk des Soziologen und Sinologen Karl Wittfogels: Wittfogel (1957).

7 Ostrowski (1998), 86. Zur sozialen Schichtung vgl. Spuler (1965), 293-300.

8 Vgl. dazu Hobson (2004); Weatherford (2004).

9 Epstein (1996), 289. 
Die Herrschaftsbeziehungen zwischen den metropolitanen Zentren - also Sarai bzw. Venedig und Genua - und den Außenposten/Kolonien nicht nur auf der Krim entsprachen nicht dem neuzeitlichen Muster des Kolonialismus, das von Jürgen Osterhammel treffend als Relation „zwischen Kollektiven, bei welcher die fundamentalen Entscheidungen über die Lebensführung der Kolonisierten durch eine kulturell andersartige und kaum anpassungswillige Minderheit von Kolonialherren unter vorrangiger Berücksichtigung externer Interessen getroffen und tatsächlich durchgesetzt wird“, ${ }^{10}$ beschrieben worden ist. Die italienischen Stadtstaaten standen eher einem „Emporion“, also einem Netz von Handelsniederlassungen als einem „Imperium“ vor, setzten auf indirekte Herrschaft und kümmerten sich wenig um die Lebensführung der lokalen Bevölkerung in den sog. Kolonien; dies zumindest solange ihre vorrangigen Interessen - und diese waren primär wirtschaftlicher Art - nicht berührt wurden. ${ }^{11}$ Städte wie Caffa erfreuten sich somit einer gewissen Unabhängigkeit, welche zum Teil soweit ging, dass diese eigene Münzen prägen konnten. Überhaupt muss der Anteil privater, lokaler Entrepreneure im Schwarzmeerhandel in dieser Zeit betont werden. ${ }^{12}$ Ein Merkmal der mongolischen Herrschaftsausübung wiederum war die schnelle Akkulturierung an die autochthonen Bevölkerungen der eroberten Gebiete sowie ein ausgefeiltes Tribut- und Steuersystem. ${ }^{13}$ In dieses waren im Übrigen auch die italienischen Händler einbezogen. ${ }^{14}$ Die Androhung und gegebenenfalls auch die Ausübung von Gewalt als Mittel zur Durchsetzung eigener Interessen spielte bei den mongolischen und den italienischen Akteuren durchaus eine Rolle, war aber auch bei der Horde eher letzter Behelf denn Mittel erster Wahl. Gleichwohl gab es zwischen den Kolonien der Seemächte auf der Krim und der Horde auch während der Pax Mongolica Konflikte, und zum Teil entluden diese sich gewaltsam; so z. B. ab 1307, als Chan Tohtu (Tutay; um 1270-1312/13) die in Sarai befindlichen italienischen Händler erst verhaften ließ, dann nach Soldaia/Sudak zurückschickte

10 Osterhammel (2003), 21.

11 Vgl. Karsten (2012), $41 \mathrm{f}$.

12 Di Cosmo (2010), 99. Indikator für eine gewisse Selbstverwaltung der Kolonien ist im Schwarzmeerraum z.B. die 1113 erfolgte Einrichtung einer Art Verwaltungsbüro: „Eight officials regulated navigation to Tana, Caffa and the Black Sea [...]. The office of the Crimea soon had jurisdiction over all ships sailing beyond Sicily and Maiorca.“ Dieses Officium Gazariae überwachte überdies das für den Schiffsverkehr geltende Regelwerk, so Epstein (1996), $193 \mathrm{f}$.

13 Der Mittelalterhistoriker Valentin Groebner macht zu Recht darauf aufmerksam, dass unter den Begriffen wie „Vermischung“, „Integration“ oder auch „Akkulturation“ letztlich Folgendes zu verstehen ist: „Es ist das, was ankommende Migranten mit der lokalen Bevölkerung tun und umgekehrt, Eroberer mit Unterworfenen, Sklavenbesitzer mit ihren Sklavinnen und Sklaven: kurz, Sex zwischen Menschen unterschiedlicher Herkunft.“ Groebner (2007), 432.

14 Di Cosmo (2010), 85. 
und ihre Waren beschlagnahmte. Damit nicht genug, sandte der Chan zudem Truppen nach Caffa, welche die Stadt acht Monate lang belagerten, ehe sich die BewohnerInnen mit ihren Schiffen in Sicherheit brachten, nachdem sie ihre Stadt in Brand gesetzt hatten. Erst 1316 wurde sie wieder aufgebaut. ${ }^{15}$ Auch untereinander führten die italienischen Seestädte Krieg, so z. B. den Zweiten VenezianischGenuesischen Krieg (1294-1299), in dem es nicht zuletzt auch um die Suprematie im Schwarzen Meer ging; dies war ein Konflikt, aus dem die Genuesen mittelfristig siegreich hervorgehen sollten. ${ }^{16}$

Die unter den Bedingungen der Pax Mongolica und auch in der Zeit danach gehandelten Waren waren seit der Antike mehr oder weniger die gleichen geblieben: Getreide, Pelze, Wachs, Honig, Seide, Salz, Gewürze sowie SklavInnen. ${ }^{17}$ Die Horde und die italienischen Stadtstaaten arbeiteten Hand in Hand - auch auf dem Gebiet des Menschenhandels. ${ }^{18}$ Erstere fungierte als Beschaffer der Ware Mensch, die Italiener verkauften sie dann von Caffa, Tana und anderen Kolonien am Schwarzen Meer aus weiter. Der Anteil, die Folgen und auch die Diskurse über den von christlichen Kaufleuten im Schwarzen Meer durchgeführten Handel mit der Ware Mensch sind mittlerweile gut erforscht. ${ }^{19}$ Ob der dortige Umschlag jemals das Ausmaß des transatlantischen Menschenhandels annahm, kann nicht mit Gewissheit gesagt werden, ${ }^{20}$ er war aber in jedem Fall ein bedeutender Wirtschaftsfaktor. Nach Abschluss eines Vertrags zwischen Michael VIII. Palaiologos und dem ägyptischen Sultan Baybars (ca. 1223-1277), in welchem letzterem das Recht auf den jährlichen Import von zwei Schiffsladungen ${ }^{21}$ voller Sklaven aus der Schwarzmeerregion zuerkannt wurde, entwickelte sich die ,Sparte' besonders dynamisch. Genuesen und Venezianer richteten in Caffa, Tana und Alexandria gut florierende Sklavenmärkte ein. Selbst nach dem Ende der Pax Mongolica lief das Geschäft ausgezeichnet: Zwischen 1414 und 1423 sollen allein aus Caffa 10.000 importierte Sklavinnen und Sklaven nach Venedig verkauft worden sein. ${ }^{22}$ Seit der Antike war der über das Schwarze Meer abgewickelte Menschenhandel eine sehr internationale Angelegenheit, beteiligten sich doch

15 Brătianu (1969), 262.

16 Vgl. Rösch (2000), $77 \mathrm{f}$.

17 Vgl. hierzu u. a. Hryszko (2004); Brătianu (1969), u.a. 186, der dem Sklavenhandel als Wirtschaftsfaktor in der mongolischen Gesellschaft eine größere Bedeutung zumisst als in den Feudalstaaten Europas.

18 Seit dem 10. Jahrhundert beteiligten sich auch slavische Händler in einem nicht genau feststellbaren Ausmaß am Sklavenhandel. Vgl. Fisher (1972b), 576.

19 Epstein (2001); Quirini-Popławska (2002).

20 King (2004), 116, und die dort angeführte Literatur.

21 Es konnte nicht eruiert werden, wie viele Menschen eine Schiffsladung umfasste.

22 Vgl. Fisher (1972b), 577. 
viele ethnische, kulturelle und religiöse Gruppen daran, so z.B. die seit der Renaissance in gebildeten europäischen Oberschichtskreisen so verehrten ,alten Griechen oder seit dem 10. Jahrhundert auch aus der Kiewer Rus’ stammende slavische Händler. ${ }^{23}$ In westlichen Diskursen wurde jedoch primär der muslimische Anteil an diesem globalen Geschäft hervorgehoben, worauf im Kontext des Krim-Chanats noch einmal zurückzukommen zu sein wird. ${ }^{24}$ Für die Venezianer und dies gilt auch für die Genuesen - ist aber zu konstatieren, dass diese von Beginn an „ein Volk von Sklavenhändlern“ gewesen sind. ${ }^{25}$

Auf die positiven Folgen der Pax Mongolica, die das Schwarze Meer mit den Worten Brătianus ja zur ,plaque tournante du trafic international“ gemacht hatte, ${ }^{26}$ ist bereits hingewiesen worden, verbreiteten sich doch nicht nur Waren, sondern auch Wissen. Es gab aber genauso negative Folgen - und dazu zählte die Verbreitung der Großen Pest ${ }^{27}$, welche verheerend wirkte, weite Landstriche Europas entvölkerte ${ }^{28}$ und im kollektiven Gedächtnis des Kontinents traumatische Spuren hinterlassen hat. ${ }^{29}$ Neal Ascherson fasste diese Dialektik knapp und treffend zusammen: „Die Seidenstraßen brachten Reichtum, dann aber den Tod.“" ${ }^{30}$ Über Jahrhunderte und sogar noch unter Epidemiologen unserer Zeit verband/verbindet man mit Caffa das Übergreifen der Pest nach Europa. Im Zentrum steht dabei eine Geschichte, welche in leicht abgewandelten Varianten immer wieder erzählt wird und deren Authentizität stark angezweifelt werden muss. Sie sagt in jedem Fall einiges über das europäische MuslimInnen-Bild aus: Bei einer der zeitweisen Auseinandersetzungen zwischen der Horde und den Genuesen im Jahr 1347 hätten die mongolischen Belagerer die Körper von Pesttoten aus ihrem Heer in die überwiegend christliche Stadt katapultiert, um die widerständigen Bürger des ausgezeichnet befestigten Caffas zu schwächen. In der westlichen Forschung wird schon seit längerer Zeit vermutet, dass diese auf den aus Piacenza stammenden Rechtsgelehrten Gabriel de Mussis (ca. 1280 - ca. 1356) zurückgehende Geschichte nicht überzeugen kann. Mittlerweile gilt es als gesi-

\section{Fisher (1972b), 576.}

24 Dazu detaillierter für diesen späteren Zeitraum Kizilov (2007).

25 Rösch (2000), 138.

26 Brătianu (1969), 225.

27 Dazu Bernstein (2009), zur Großen Pest $138 \mathrm{f}$.

28 Zur Einführung Fouquet u. Zeilinger (2011), zur Großen Pest besonders 107-110. Über die Opferzahl herrscht in der Forschung keine Einigkeit: Brătianu (1969), 237, geht beispielsweise von ca. einem Drittel der damaligen europäischen Bevölkerung aus, was für 20 bis 25 Millionen Tote spräche. Weitaus höher die Schätzung bei Benedictow (2004), 380f., der sechzig Prozent für wahrscheinlich hält.

29 Meier M. (2005).

30 Ascherson (1996), 152. 
chert, dass de Mussis kein Augenzeuge der Ereignisse von Caffa gewesen sei - und diesen Ort niemals besucht habe, wie man lange fälschlicherweise annahm. Die Pest hat vermutlich ,schon auf andere Weise [...] auf die belagerte Stadt übergegriffen“, durch infizierte Ratten oder Flöhe beispielsweise, die in direkten Kontakt mit den BewohnerInnen der Stadt gekommen sein mussten. ${ }^{31}$ In einer medizinischen Zeitschrift wurde vor einigen Jahren nämlich festgehalten, dass der in dieser Legende beschriebene Übertragungsweg der Yersinia pestis, wie der lateinische Name der Pest lautet, unrealistisch ist, denn infizierte Flöhe verlassen tote Körper, die somit selbst nicht mehr ansteckend seien. ${ }^{32}$ Gerne wurde die Katapulterzählung aber als eine frühe Form bakteriologischer Kriegsführung interpretiert, erdacht und durchgeführt von den sinistren Angehörigen der asiatischen Horde. ${ }^{33}$ So oder so - von der Krim aus verbreitete sich die Epidemie mit großer Geschwindigkeit und den bekannten, schrecklichen humanitären Folgen. Wirtschaftlich fiel die Bilanz der Pestfolgen für Niederlassungen wie Tana oder Caffa dennoch gemischt aus: Durch die Auseinandersetzungen mit der Goldenen Horde unter der Herrschaft des Chans Ğani Beg (Dschani Beg; ?-1357; Chan 1342-1352) und die Entvölkerung zahlreicher Städte im Schwarzmeerraum durch die Pandemie verschlechterte sich die Lage erst einmal; ${ }^{34}$ da durch den Schwarzen Tod aber europaweit ein großer Mangel an Arbeitskräften entstanden war, verteuerte sich alsdann die Ware Mensch - wovon die italienischen Kolonien als Umschlagplatz für Sklavinnen und Sklaven profitierten.

Albrecht und Herdick stellen fest, dass sich Genua erst dann - in der zweiten Hälfte des 14. Jahrhunderts - von einem „im Wesentlichen ökonomischen zu einem bedeutenden politischen Faktor auf der Krim zu entwickeln“ begann. ${ }^{35} 1365$ eroberte Genua beispielsweise das bislang vorwiegend von Venedig beherrschte Soldaia/Sudak, womit dem langjährigen Konkurrenten ein erheblicher Schlag versetzt wurde. In dem den Vierten genuesisch-venezianischen Krieg beendenden Frieden von Turin (1381) wurde Venedig sogar für zwei Jahre verboten, Tana anzulaufen. Es zeigte sich also: „Genua aber war mächtiger“36 - als Venedig.

Parallel veränderte sich auch die Lage eines anderen Akteurs, denn nach dem Tod des Chans Ğani Beg zeichnete sich der allmähliche Niedergang der Goldenen

31 Bulst (1979), hier 46.

32 Gerste (2004).

33 Vgl. z. B. Derbes (1966). Dito bei Brătianu (1969), 244, über „[c]ette formule de guerre microbienne“.

34 Ascherson (1996), 153, gibt an, dass zwischen Dezember 1347 bis September 1348 drei Viertel der europäischen Bevölkerung in den dortigen Kolonien starben.

35 Albrecht u. Herdick (2013), 41.

36 So auch der Titel einer populärwissenschaftlichen Publikation: Kurowski (1986). 
Horde ab, wurden die Partikularinteressen lokaler Emire doch immer stärker. Zwar versuchte sich der Emir Mamāi (1335-1380), der sich am unteren Don und eben auf der Krim eine Machtbasis hatte aufbauen können, noch einmal an der Einigung der auseinanderstrebenden Gebiete, scheiterte damit aber: Der Versuch, die mongolische Dominanz in der Kiewer Rus', deren Gebiete ja seit Mitte des 13. Jahrhunderts fast vollständig tributpflichtig waren, wieder herzustellen, kulminierte in der im russischen kollektiven Gedächtnis bis in unsere Zeit so berühmten Schlacht auf dem Kulikovo pole (Schnepfenfeld) im Jahr 1380. Mamāis Truppen verloren diese bekanntlich, was in der Rückschau als der Anfang vom Ende der Mongolenherrschaft über die Rus' interpretiert wurde. Und sogar mehr als das: Wenn man denn Lev N. Gumilëv (1912-1992) folgen will, Sohn der großen russischen Dichterin Anna Achmatova und ein den sog. Eurasiern zuneigender Historiker, markiert diese Schlacht die Geburt der russischen Nation: „Leute aus Suzdal', Vladimir, Rostov, Pskov gingen auf das Feld von Kulikovo, um als Vertreter ihrer Fürstentümer zu kämpfen, aber sie kehrten von dort als Russen zurück. “37 Er sprach der Schlacht deshalb weltgeschichtliche Bedeutung zu. Für die Geschichte der Krim ist vor allen Dingen Folgendes von Relevanz: Im Vorfeld des Waffenganges gegen die Rus' hatte es eine Verbesserung des mongolisch-genuesischen Verhältnisses gegeben, welches ja in der ersten Hälfte des Jahrhunderts angespannt gewesen war. Folgerichtig suchte - und fand - Mamāi nach der Niederlage auf dem Kulikovo pole Zuflucht hinter den Mauern Caffas, wo er allerdings einem Giftanschlag erlag. ${ }^{38}$

Der Chan der Goldenen Horde Toktamış (Toktamisch/Toqtamisch/Tohtamyš; ?-1406/1407; Chan der Goldenen Horde 1380-1395) führte, obgleich er den Sonderwegen Mamāis ablehnend gegenüberstand, die von diesem wieder aufgenommene Politik der Kooperation mit den Genuesen - zum Nachteil der ohnehin schon im Schwarzen Meer ins Hintertreffen gelangten Venezianer - fort. Genua dominierte fortan letztlich die ganze südliche Krim-Küste einschließlich des Azovschen Meeres und der Halbinsel Taman. Allerdings wurden viele Gebiete in der Region in die immer stärkeren Konflikte zwischen der Goldenen Horde und dem Timuriden-Reich unter Timur (Lenk, der Lahme;1336-1405) hineingezogen. ${ }^{39}$ Dessen militärischen Erfolge, Talente, aber auch seine Gewaltbereitschaft werden häufig mit denen des großen Mongolen-Chans Činggis Qayan verglichen. ${ }^{40} 1395$

37 Gumilëv (1997), 173.

38 Spuler (1965), 120.

39 Nagel (1993).

40 So z. B. Soucek (2000), 123. Das Timuriden-Reich existierte zwischen 1370 und 1507, dessen Kerngebiete umfassten in etwa die heutigen Staaten Afghanistan, Iran und Usbekistan. Hauptstadt war anfangs Samarkand, später Herat. 
zerstörten dessen nach Westen vordringenden Truppen Tana und auch Teile der Krim. 